\title{
Theory of two-fluid metallicity in superconducting FeSe at high pressure
}

\author{
L. Craco ${ }^{1}$ and S. Leoni ${ }^{2}$ \\ ${ }^{1}$ Instituto de Física, Universidade Federal de Mato Grosso, 78060-900 Cuiabá, MT, Brazil \\ ${ }^{2}$ School of Chemistry, Cardiff University, Cardiff CF10 3AT, United Kingdom
}

(Received 18 February 2019; revised manuscript received 21 August 2019; published 3 September 2019)

\begin{abstract}
We compute the two-fluid resistivity of a compressed FeSe superconductor, treating electronic correlations within density functional dynamical mean-field theory. Results for the emergent strange and bad metal behavior shows good theory-experiment agreement within the mixed tetragonal and pseudohexagonal structural phases at pressures around $8.1 \mathrm{GPa}$. Our findings call for more studies on unconventional high-temperature (high$T_{c}$ ) superconductors to unearth the consequences of selective Mott localization as the prominent candidate in governing their strange, $T$-linear resistivity.
\end{abstract}

DOI: 10.1103/PhysRevB.100.121101

\section{INTRODUCTION}

The normal state of a variety of correlated electron systems often falls into the strange [1] and bad [2] metal category, where the electrical resistivity varies linearly with temperature $\left[\rho_{\mathrm{dc}}(T)=\rho_{0}+A T\right]$ as $T \rightarrow 0$ [3]. This generic property, together with other experimental evidence such as the absence of Drude peak in optics, an incoherently broad line shape in spectroscopy, and a strongly $T$-dependent Hall effect, represent significant deviations from the conventional Fermiliquid (FL) picture of ordinary, good metals. Although the fundamental origin for this anomalous non-FL behavior [4] is under debate [5], the $T$-linear resistivity is often associated with quantum criticality [6]. On general grounds the strange metal phase exhibits a nonsaturating, $T$-linear resistivity that extends well beyond the Mott-Ioffe-Regel limit [7] at higher temperatures, due to vanishing quasiparticle excitations [8]. Examples of materials showing pseudogap features or the absence of electron quasiparticles include $\mathrm{Cu}$ oxide [3] and heavy fermion materials at or close to a quantum critical point [4]. Remarkably as well within the strongly correlated strange metal context is the $T$-linear resistivity recently seen in magicangle twisted bilayer graphene [9] as well as in high- $T_{c}$ superconducting $\mathrm{FeSe}_{1-x} \mathrm{~S}_{x}$ systems [10-13] at high pressures.

In spite of its seemingly simplest chemical form, FeSe is one of the most studied members of the Fe-superconducting family, displaying different coexisting structural and magnetic phases [12,14-17]. Below 6-7 GPa, FeSe has a tetragonal structure (space group $P 4 / \mathrm{nmm}$ ) at room temperature which becomes a slightly distorted orthorhombic structure $(\mathrm{Cmma})$ upon cooling [16]. The low-temperature orthorhombic phase, denoted as ortho-I [16], is characterized by an orbital nematic order followed by a magnetostructural transition driven by stripe-type spin fluctuations $[18,19]$. Experimental data show evidence that antiferromagnetic (AF) order exists at least up to $6 \mathrm{GPa}$ [16]. Finally, above $7 \mathrm{GPa}$, a tetragonal to pseudohexagonal (ortho-II) structural phase transition [16,20] is observed.

The superconducting $T_{c}$ in FeSe shows rich pressure dependence. After a small initial increase, $T_{c}$ reduces at the onset of
AF order at pressure below $2 \mathrm{GPa}$ : This low-pressure behavior has been attributed to a reduction in the density of states (DOS) owing to Fermi-surface reconstruction [21]. Increasing pressure enhances both $\mathrm{AF}$ and superconducting (SC) orders, with the Néel temperature $T_{N}$ reaching 45-55 K [11] at $4.2 \mathrm{GPa}$ and $T_{c} \approx 20 \mathrm{~K}$ [22]. Above this pressure, magnetic order decreases and an optimal $T_{c}$ is achieved when the longrange $\mathrm{AF}$ order vanishes at pressures close to $6 \mathrm{GPa}[11,14]$. The decrease of $T_{c}$ above this pressure coincides with the appearance of a mixed ortho-I plus ortho-II structural phase at low temperatures [16], and it is consistent with the coexistence of SC (tetragonal) and non-SC (pseudohexagonal) electronic fluids at high pressures [19]. (Evidence from high-pressure transport data $[11,14]$ indicates that the ortho-II phase does not support SC). Such a complex pressure-temperature phase diagram [12,22], which is partially reshaped upon substitution of $\mathrm{S}$ for Se [13], manifests the nontrivial interplay between electronic, orbital, spin, and structural degrees of freedom which defines the SC phase boundary in $11 \mathrm{Fe}$-chalcogenide $\mathrm{FeSe}_{1-x} \mathrm{~S}_{x}$ superconductors.

The $11 \mathrm{Fe}$-chalcogenide superconductors offer a good platform for the study of orbital-selective (OS) electronic reconstruction [23,24] as well as the $T$-linear resistivity [10-13] of strange metals. Experimentally, at a pressure range between 7.8 and $8.8 \mathrm{GPa}$, the AF order in FeSe no longer competes with superconductivity and the resistivity displays an almost perfect $T$-linear dependence in a wide temperature range from room temperature down to $T_{c}$ [10-12]. Nearly linear dependence has also been report in $\mathrm{FeSe}_{1-x} \mathrm{~S}_{x}$ systems at similar pressure conditions [13], implying a common mechanism for the strange metal behavior. The most fundamental questions here are as follows: Is the $T$-linear resistivity limited to layered materials or is it more universal in spite of many different slopes [7,9] found in experiment? How does strange, bad metallicity in FeSe arise under pressure? In this Rapid Communication we aim to answer this latter question, providing a microscopic description of the anomalous transport seen in a compressed FeSe superconductor. We reveal a surprising contrast between coherent and incoherent phenomena induced by multiorbital $(\mathrm{MO})$ electron-electron interactions on the 
coexisting (ortho-I and ortho-II) structural phases at high pressures. Needless to say, a proper microscopic description of strange and bad metallic states is a basic prerequisite for understanding how exotic are the electronic states of $11 \mathrm{Fe}-$ chalcogenide superconductors at high pressures.

\section{THEORY AND RESULTS}

Theoretical attempts toward a realistic description of strange, bad metals involves going beyond traditional FL concepts of the low-energy electronic coherence of good metals. With this in mind, and focusing on stoichiometric FeSe, here we carried out a comprehensive numerical study for tetragonal and pseudohexagonal $\mathrm{FeSe}$, providing theoretical evidence of a distinct orbital-selective two-fluid metal in a highly compressed FeSe superconductor. We use dynamical meanfield theory (DMFT) in conjunction with the local density approximation (LDA + DMFT) [25] to address this issue of fundamental importance.

Within LDA, the one-electron part of the many-body Hamiltonian for $\mathrm{FeSe}$ is $H_{0}=\sum_{\mathbf{k} a \sigma} \epsilon_{a}(\mathbf{k}) c_{\mathbf{k} a \sigma}^{\dagger} c_{\mathbf{k} a \sigma}$, where $a=x^{2}-y^{2}, 3 z^{2}-r^{2}, x z, y z, x y$ label the diagonalized, five $3 d$ bands of iron. In light of the sizable correlation effects in $11 \mathrm{Fe}$ chalcogenides [23,24,26-29], however, full MO Coulomb interactions must be included to describe the onset of correlated spectral functions. These constitute the interaction term, which reads $H_{\text {int }}=U \sum_{i, a} n_{i a \uparrow} n_{i a \downarrow}+$

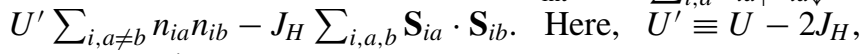
with $U\left(U^{\prime}\right)$ being the intraorbital (interorbital) Coulomb repulsion and $J_{H}$ is the Hund's rule coupling. For FeSe, the five $3 d$ bands crossing the Fermi energy were obtained using the linear-muffin-tin orbital (LMTO) [30] scheme within the LDA. We evaluate the many-particle Green's functions $\left[G_{a, \sigma}(\mathbf{k}, \omega)\right]$ of the MO Hamiltonian $H=H_{0}+H_{\text {int }}$ within LDA + DMFT [25], using MO iterated perturbation theory (MO-IPT) as the impurity solver [31]. The DMFT solution involves replacing the lattice model by a self-consistently embedded MO-Anderson impurity model, and the selfconsistency condition requiring the local impurity Green's function to be equal to the local Green's function for the lattice. The full set of equations for the MO case can be found in Ref. [31] so we do not repeat the equations here. This real frequency perturbative ansatz has a proven record of good semiquantitative agreements with experiment for a range of $d$ - and $s$-band materials, and as shown below it gives results in qualitative accord with numerical exact methods [24].

Previously, Skornyakov et al. [24] have employed a fully charge self-consistent density functional theory plus DMFT (DFT + DMFT) scheme to perform direct structural optimization and band-structure calculations of tetragonal FeSe at ambient and high-pressure (10 GPa) conditions, showing a topological change in the Fermi surface (Lifshitz transition) of compressed FeSe. Based on their results, at about $10 \mathrm{GPa}$, FeSe should be regarded as a moderately correlated metal with coherent FL quasiparticles spanning at the Fermi level $E_{F}$. Although transport data exist [11], this pressure is slightly above the regime where the strange metal phase is clearly observed [12,13]. Noteworthy, in their work, Skornyakov et al. have used $U=3.5 \mathrm{eV}$ and $J_{H}=0.85 \mathrm{eV}$ as representative model parameters at ambient and high-pressure conditions. As

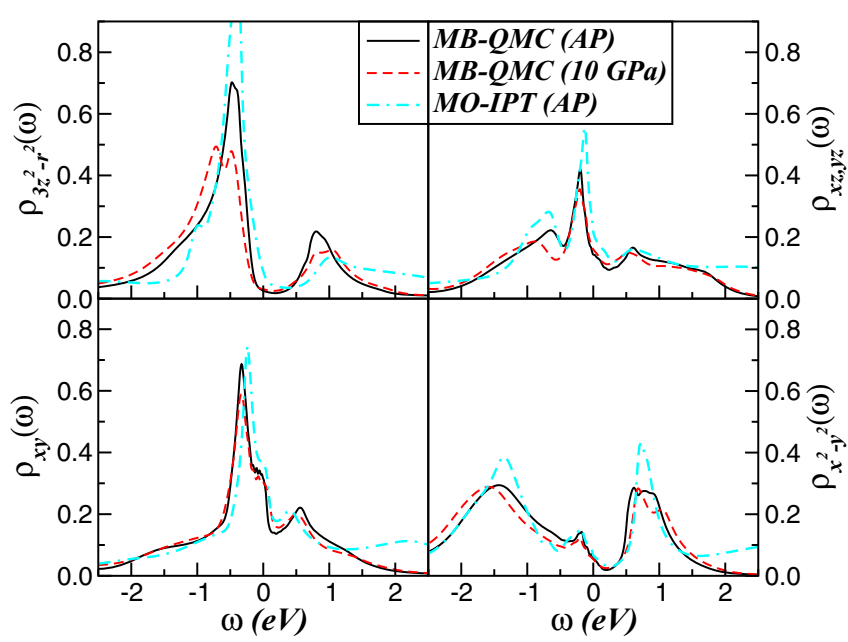

FIG. 1. Orbital-resolved density of states (DOS) for the Fe $d$ orbitals of tetragonal FeSe, computed using the multiband, continuoustime quantum Monte Carlo (MB-QMC) [24] and multiorbital iterated perturbation theory (MO-IPT) [29] impurity solver of DMFT. As seen, MO-IPT successfully reproduces the main features of numerically exact MB-QMC for a tetragonal FeSe superconductor, regardless of the screening effects due to charge self-consistent calculations. In Ref. [24], MB-QMC calculations at ambient and high pressures were performed at $T=290 \mathrm{~K}$. Here, all LDA + DMFT (MO-IPT) spectral functions are computed at zero temperature.

can be seen in Fig. 1, within this set of parameters a predominant contribution of electronic states at $E_{F}$ arises from $t_{2 g}$-like $(x z, y z, x y)$ electrons, while the $e_{g}\left(3 z^{2}-r^{2}, x^{2}-y^{2}\right)$ orbital sector shows depleted (band gapped) electronic states at $E_{F}$. Interestingly, upon compression no substantial spectral weight transfer (SWT) was found in this multiband, continuoustime quantum Monte Carlo (MB-QMC) study. This DFT + DMFT study reveals a weak increase in the electron bandwidth in compressed FeSe, meaning that the overall line shape of the correlated spectral functions should show only a weak pressure dependence within the tetragonal structural phase.

To make progress, in Fig. 1 we display our self-consistent LDA + DMFT (MO-IPT) result computed using $U=2.0 \mathrm{eV}$ and $J_{H}=0.75 \mathrm{eV}$ [29]. As a major finding, our calculated MO-IPT DOS shows qualitatively good agreement with MBQMC results. (We note, however, that our $3 z^{2}-r^{2}$ DOS was shifted downwards by $0.18 \mathrm{eV}$ to coincide with the orbitalenergy levels of MO-IPT and MB-QMC). Taken together with earlier studies [32], this direct comparison testifies to the reliability of the MO-IPT scheme, providing a consistent explanation for the most relevant correlation fingerprints seen in the occupied and unoccupied electronic states within the energy range relevant to transport and spectroscopy experiments. More importantly, the clearly visible similarities obtained using different $U$ values seem to suggest that the incorporation of Se $4 p$ orbitals in the multiband and many-particle problem of tetragonal FeSe leads to a $d$-band model with an effective bandwidth that is enhanced relative to the starting bare bandwidth $(W)$ of the Fe-3d shell. SWT from low to high binding energies is expected to enhance the $p d$ hybridization, leading to an effective screened Coulomb interaction in the correlated 


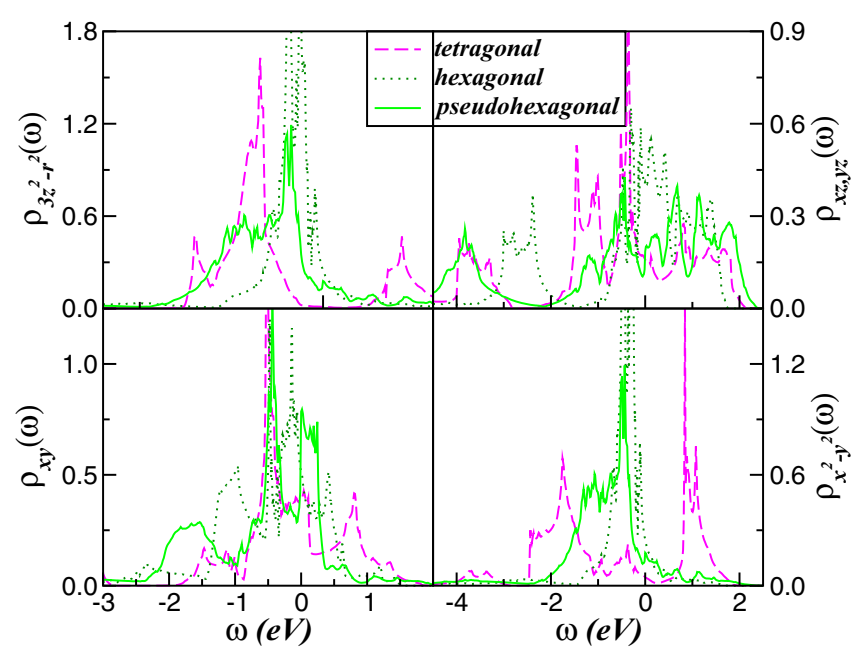

FIG. 2. Orbital-resolved LDA DOS for the Fe $d$ orbitals of tetragonal, hexagonal, and high-pressure (pseudohexagonal) FeSe computed using the LMTO method. Notice that all $d$ bands span over the Fermi level. This shows that the electronic states relevant to $\mathrm{Fe}$ chalcogenides are $\mathrm{Fe} d$ states. A large spectral weight transfer induced by one-particle band broadening is visible in pseudohexagonal FeSe.

subspace $[33,34]$. Future fully charge self-consistent DFT + DMFT calculations should consider intrinsic pd screening effects as well as the importance of incorporating within the same theoretical framework stronger electronic correlations in all active band states [34].

In order to get insights into the electronic structure evolution of FeSe at low- and high-pressure phases, in Fig. 2 we compare the bare one-electron band structure of tetragonal [29], hexagonal [28], and pseudohexagonal (with the experimentally determined crystal structure parameters at $8.1 \mathrm{GPa}$ ) [16] FeSe computed using the LMTO scheme in the atomic sphere approximation [30]. The structural-induced electronic anisotropies are clearly manifested in our results. The $e_{g}$ orbitals, which are almost gapped at $E_{F}$ in the tetragonal phase, go over into highly polarized, narrow bands centered at $-1.1 \mathrm{eV}$ in hexagonal FeSe at ambient pressure. Differently to what is found within the tetragonal structural phase, these orbitals are twofold degenerated in the perfect hexagonal phase. However, as expected and similar to what happens in nematic (ortho-I) FeSe [35], the distorted ortho-II structure lifts the $x z, y z$ orbital degeneracy (not shown), but the overall line shape in this ferro-orbital ordered state is very similar to the average $x z, y z$ DOS of Fig. 2. Additionally, due to the smaller volume of the pseudohexagonal structure relative to the ambient pressure one, the LDA bandwidth is considerably enhanced in the ortho-II phase at $8.1 \mathrm{GPa}$. Also interesting is the downshift of the sharp peak at $0.14 \mathrm{eV}$ binding energy seen in the $x y$ electronic structure, which coincides almost perfectly with that of tetragonal FeSe at $0.51 \mathrm{eV}$ below $E_{F}$. Thus, differently to what has been proposed for tetragonal FeSe [24], our results demonstrate the electronic structure of hexagonal FeSe is sensitive to the structure [12], and considerable changes should be expected in the electronic structure of hexagonal FeSe at high pressure.

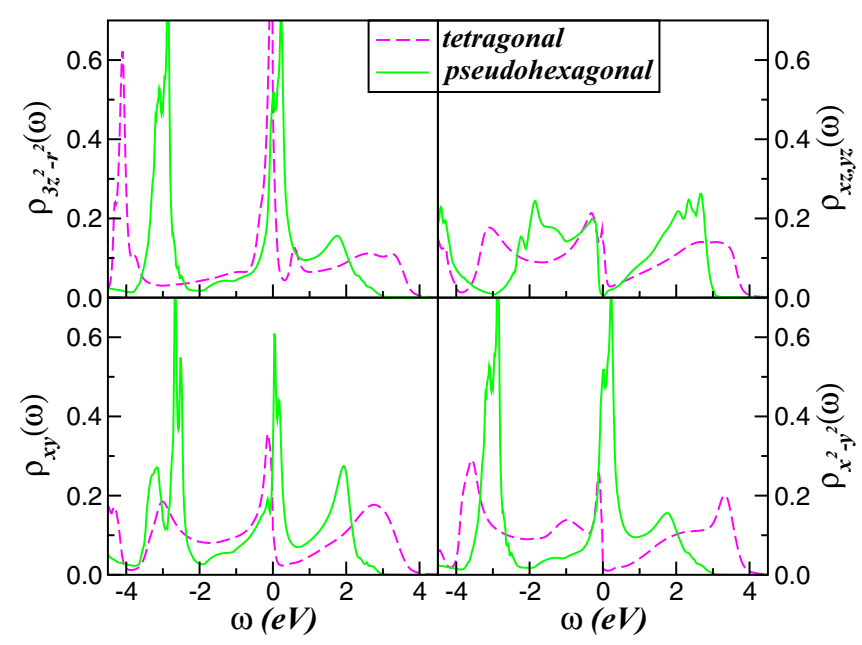

FIG. 3. Orbital-resolved Fe-3d DOS of compressed, tetragonal (dashed line), and pseudohexagonal (solid line) FeSe. Notice the modification of the LDA + DMFT spectra with coexisting Fermiliquid quasiparticles and quasi- or totally-Mott localized electronic states at low energies.

To obtain detailed information on the evolution of the correlated electronic structure from a tetragonal to pressurized, pseudohexagonal phase [16,20], in Fig. 3 we show our $\mathrm{LDA}+$ DMFT results obtained within the the $\mathrm{Fe}^{2+}$ oxidation state. It is worth mentioning here that starting with the five Fe $3 d$ orbitals shown in Fig. 2, in earlier studies we have derived the correlated electronic structure of a tetragonal FeSe superconductor at ambient pressure [29]. A good theoryexperiment comparison of the one-particle spectral function in the normal [29] and superconducting state [35] as well as with transport data [26] were found for $U=4.0 \mathrm{eV}$ and $J_{H}=$ $0.7 \mathrm{eV}$ : This parameter choice is consistent with other theoretical works, showing that tetragonal Fe-based superconductors are correlated electron systems with an $U / W$ ratio close to unity [36]. However, given the non-negligible $d$-electron band broadening [24] in this work, we chose $U=3.5 \mathrm{eV}$ as the effective Coulomb parameter for compressed tetragonal and pseudohexagonal FeSe.

As seen in Fig. 3, electronic correlations lead to interesting modifications of the LDA spectra: In both phases the manybody spectra describe an OS system with orbital-dependent low-energy coherent (FL), incoherent (non-FL), and Mott localized features on different orbitals. Relevant as well are the (upper and lower) Hubbard bands, which are more pronounced in the pseudohexagonal phase due to intrinsic band narrowing, or a higher $U / W$ ratio of effective local Coulomb correlation effects. Our LDA + DMFT results unveil that strong local moments originating from Hubbard bands coexist with low-energy, itinerant $\left(3 z^{2}-r^{2}, x y, x^{2}-y^{2}\right)$ and Mott localized $(x z, y z)$ electronic states in ortho-II FeSe at high pressures. Interestingly, the fact that these local moments do not order in the ortho-II phase $[13,22]$ can be taken as an indication of enhanced spin fluctuations [12] in FeSe at pressures where the strange metal phase is seen in experiments [10-13].

In Fig. 4, we show the orbital-resolved self-energy imaginary $\left[\operatorname{Im} \Sigma_{a}(\omega)\right]$ and real $\left[\operatorname{Re} \Sigma_{a}(\omega)\right]$ parts computed using 


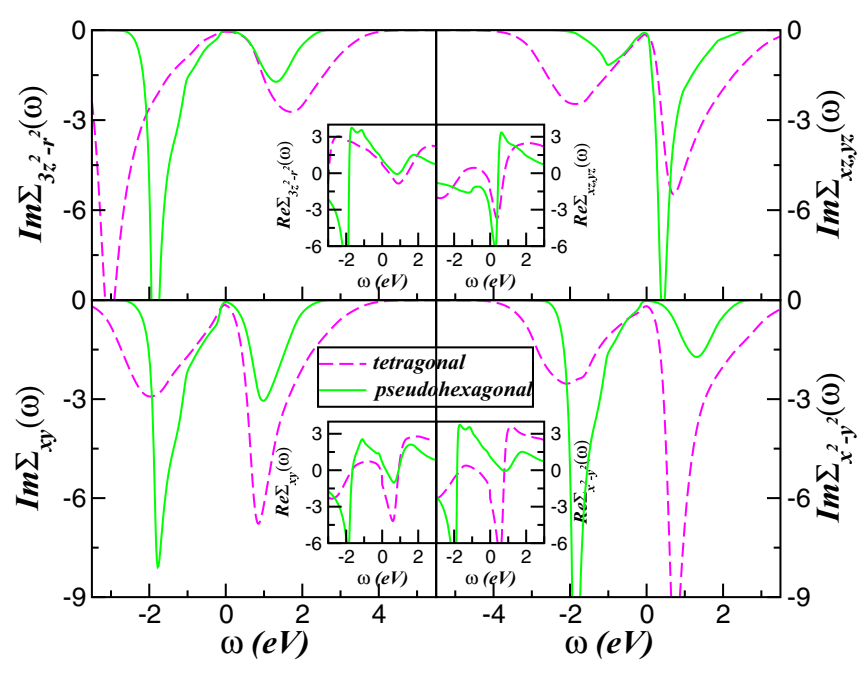

FIG. 4. Orbital-resolved self-energy imaginary (main panels) and real (insets) parts for the $\mathrm{Fe}-3 d$ orbitals of compressed, tetragonal (dashed line), and pseudohexagonal (solid line) FeSe. Notice the sharp poles in the self-energy real and imaginary parts near $E_{F}$ for the Mott localized $x z, y z$ orbital sector of pseudohexagonal FeSe.

$U=3.5 \mathrm{eV}$. A strong energy dependence of the LDA + DMFT (MO-IPT) self-energies is clearly seen: This provides a microscopic basis for rationalizing the electron correlation effects $[28,29,34]$ and the nature of bad metallic behavior in 11 Fe-chalcogenide systems [23,26]. In Fig. 4 we identify incoherent and coherent behavior on different orbitals as well as the pole generating the proximity to Mott localization in the $x z, y z$ (pseudohexagonal) and $x^{2}-y^{2}$ (tetragonal) orbital sectors. As seen, due to the interplay between MO electronelectron interactions and structural-dependent SWT, a pole structure responsible for the strange, bad metal state and proximity to orbital-selective Mott localization evolves near $E_{F}$ in compressed FeSe. We predict that similar features as in Fig. 4 would be seen in scattering rates $\tau^{-1}(\omega) \simeq \operatorname{Im} \Sigma(\omega)$ [37], and future angle-resolved photoemission spectroscopy (ARPES) studies are called for to corroborate our prediction. Moreover, since in DMFT the self-energy is momentum independent, the quasiparticle residue $Z$ directly yields the effective electron mass enhancement [38] $\frac{m_{a}^{\star}}{m}=\frac{1}{Z_{a}}=\left(1-\frac{\partial \operatorname{Re} \Sigma_{a}(\omega)}{\partial \omega}\right)_{\omega=0}$, where $m$ is the bare electron mass. Thus, from the slope of the self-energy real part in Fig. 4, we obtain $Z_{a}$, which translates into an orbital-dependent effective mass enhancement $\left(\frac{m_{3 z^{2}-r^{2}}^{\star}}{m}, \frac{m_{x z, y z}^{\star}}{m}, \frac{m_{x y}^{\star}}{m}, \frac{m_{x^{2}-y^{2}}^{\star}}{m}\right)$ of $(5.3,19.7,27.0,22.9)$ for tetragonal and $(3.1,11.8,4.8,3.1)$ for pseudohexagonal FeSe superconductors. Observation of large $\frac{m^{\star}}{m}$ values in ARPES experiments [39] would attest to the dynamical many-particle correlation effects in the normal state of 11 Fe chalcogenides at ambient and high-pressure conditions. Finally, it is worth noting that deviations from the $\omega^{2}$ dependence of a canonical FL, with a sublinear $\omega$ dependence of marginal Fermi liquids [40], is also seen in Fig. 4. A similar self-energy behavior as in Fig. 4, with sublinear and diverging energy dependences, was also found in earlier studies [23,41], implying a common scenario of a correlation-induced electronic reconstruction in

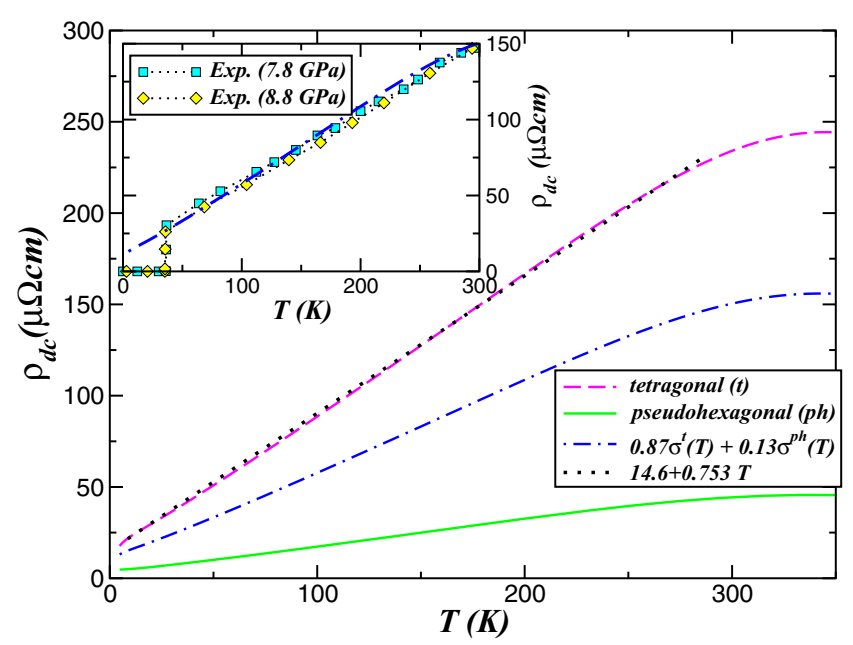

FIG. 5. Resistivity vs temperature (normalized to coincide the mixed phase with experiment) for compressed, tetragonal (dashed line), pseudohexagonal (solid line), and FeSe polymorph (dotteddashed line). Notice the almost perfect $T$-linear dependence up to $270 \mathrm{~K}$ for tetragonal $\mathrm{FeSe}$ as compared to the linear fit (doted line), followed by a maximum in $\rho_{\mathrm{dc}}(T)$ at temperatures close to $340 \mathrm{~K}$. Inset: $T$ dependence of zero-field resistivity curves [12] at high-pressure conditions, showing similar linear behavior. Qualitative good agreement between the LDA + DMFT $(U=3.5 \mathrm{eV})$ resistivity for the mixed phase and the experimental data [12] is visible, confirming that tetragonal and pseudohexagonal structural phases coexist [15] in this pressure range.

Fe chalcogenides within the LDA + DMFT (MO-IPT) treatment.

On general grounds, the quasicomplete (complete) manybody localization of the $d_{x z, y z, x y, x^{2}-y^{2}}\left(d_{x z, y z}\right)$ states in the tetragonal (pseudohexagonal) phase implies that these orbitals now act as a distinct source of local disorder in the two electronic fluids of high-pressure FeSe. With $U^{\prime}=2.1 \mathrm{eV}$, this implies that a dynamical locally coupled scattering potential arising from OS Mott physics exists in the two structural phases of compressed FeSe. Remarkably, such behavior results from strong scattering between effectively Mott localized and itinerant components of the full DMFT matrix propagators. These two-particle collective phenomena are intimately linked to double-exchange [42] physics (where localized spins of magnetic ions are coupled to mobile electrons) [43] and, as shown below, they are responsible for the strange metal behavior seen in compressed FeSe. We note, however, that orbital-selective Mott physics has also been proposed earlier for ambient pressure Fe-chalcogenide and Fe-pnictide systems [44] and discussed on phenomenological grounds in the context of 122 Fe pnictides [45].

Turning our attention to transport properties, in Fig. 5 we compare the obtained electrical resistivity $\left[\rho_{\mathrm{dc}}(T)=1 / \sigma(T)\right]$ of compressed tetragonal and pseudohexagonal FeSe, computed using the LDA + DMFT orbital-resolved spectral functions (Fig. 3) in the electronic conductivity $\sigma(T)$ [46]. Various features immediately stand out. First, $\rho_{\mathrm{dc}}(T)$ shows larger values within the tetragonal phase, in accord with the OS localization shown above. Interestingly, our result for tetragonal FeSe displays a nearly perfect $T$-linear-like form [with 
$A=d \rho_{\mathrm{dc}}(T) / d T=0.753 \mu \Omega \mathrm{cm} \mathrm{K}^{-1}$, a value close to $0.7 \pm$ $0.01 \mu \Omega \mathrm{cm} \mathrm{K}^{-1}$ found in $\mathrm{SrFe}_{2}\left(\mathrm{As}_{0.7} \mathrm{P}_{0.3}\right)_{2}$ or to $0.78 \pm$ $0.001 \mu \Omega \mathrm{cm} \mathrm{K}^{-1}$ for $\mathrm{Bi}_{2} \mathrm{Sr}_{2} \mathrm{CaCu}_{2} \mathrm{O}_{8.27}$ ] [7], characteristic of strange metals up to approximately $270 \mathrm{~K}$, followed by a maximum in the resistivity profile. Albeit at different temperatures, similar bad metal behavior characterized by a maximum in $\rho_{\mathrm{dc}}(T)$ is seen in tetragonal $\mathrm{FeSe}$ at ambient pressure [17]. The fact that $\rho_{\mathrm{dc}}(T)$ is almost linear in experiment [12] implies a weak pseudohexagonal electronic contribution in compressed FeSe at pressures up to $8.8 \mathrm{GPa}$. From Fig. 5, it is clear that $\rho_{\mathrm{dc}}(T)$ exhibits qualitatively distinct behavior in the two structural phases. Importantly, as shown in the inset, the detailed $T$ dependence for the mixed phase (tetragonal plus hexagonal phases) $[15,16]$ resembles the one seen in experiment [12], indicating a two-component electronic fluid at pressure close to $8.1 \mathrm{GPa}$. (Noteworthy, using the molar percentage obtained from experimental refinements [15], in our description we assume that the two-channel conductivities are additive [47] within the mixed phase of compressed FeSe). The observation of similar linear-in- $T$ features at $10 \mathrm{GPa}$ [11] represents additional experimental evidence of the correctness of our correlated, two-fluid scenario for compressed FeSe. We emphasize, however, that a correlated metal with purely coherent quasiparticle behavior at $E_{F}$ [24] would give $T$ dependences and resistivity values that would be in conflict with the extant data [11] at $10 \mathrm{GPa}$. As a result, we predict that destroying superconductivity by perturbations such as magnetic field, chemical substitution, or under different crystal growth conditions will reveal a first-order structural phase transition in FeSe at pressures between 10 and $12 \mathrm{GPa}$. Furthermore, a strongly incoherent electronic structure reconstruction acting as the precursor to saturated metal is expected to be seen in the pressure range between $12 \mathrm{GPa}$ [11] and $15 \mathrm{GPa}$ [14]. We defer this latter transport and structural phase study, however, to a separate work.

\section{CONCLUSION}

In summary, we investigate the electronic structure of compressed, tetragonal, and pseudohexagonal $\mathrm{FeSe}$ within
LDA + DMFT with sizable [24] multiorbital interactions. Although the main purpose of this Rapid Communication is to understand the nature of electronic transport in $\mathrm{FeSe}$ at high pressures, a major finding of the present study is that considerable changes in the electronic state are found when the $\mathrm{Fe}-3 d$ shell is released from multiband screening effects. Normal-state incoherence within the tetragonal phase, along with orbital-selective localization in the correlated electronic fluid of compressed $\mathrm{FeSe}$, both arising from local interactions, are expected to be intimately involved in the strange metal seen in $\mathrm{FeSe}_{1-x} \mathrm{~S}_{x}$ systems at pressures close to $8 \mathrm{GPa}$ [10-13]. Thermodynamically, at this pressure range, FeSe can be thought of as two metallic fluids, one which participates in the superconducting state, and another, containing a higher density of Fermi-liquid quasiparticles, which does not. As such, our two-fluid scenario should be more generally applicable to dirty superconductors, where a large portion of spectral weight does not participate in the superconducting condensate below $T_{c}$ [48]. Viewed in light of this result, our finding opens up interesting avenues for future studies on two-fluid theories of superconductivity [49].

\section{ACKNOWLEDGMENTS}

L.C.'s work is supported by CNPq (Grant No. 304035/2017-3). Acknowledgment (L.C.) is also made to the Institute for Solid State Research at IFW Dresden for hospitality. S.L. acknowledges support from the UK Research Council for using work in the paper that was undertaken by a student under Project No. EP/M50631X/1 as well as the DFG for support under the priority Project No. SPP1415 and for a personal Heisenberg grant. S.L. also thanks ARCCA Cardiff for computational resources. Via S.L.'s membership of the UK's HPC Materials Chemistry Consortium, which is funded by EPSRC (Grant No. EP/L000202), this work made use of the facilities of ARCHER, the UK's National High-Performance Computing Service, which is funded by the Office of Science and Technology through EPSRC's High End Computing Programme.
[1] P. W. Anderson, Nat. Phys. 2, 626 (2006)

[2] X. Deng, J. Mravlje, R. Žitko, M. Ferrero, G. Kotliar, and A. Georges, Phys. Rev. Lett. 110, 086401 (2013).

[3] A. Legros, S. Benhabib, W. Tabis, F. Laliberté, M. Dion, M. Lizaire, B. Vignolle, D. Vignolles, H. Raffy, Z. Z. Li, P. Auban-Senzier, N. Doiron-Leyraud, P. Fournier, D. Colson, L. Taillefer, and C. Proust, Nat. Phys. 15, 142 (2019).

[4] G. R. Stewart, Rev. Mod. Phys. 73, 797 (2001).

[5] T. Andrade, A. Krikun, K. Schalm, and J. Zaanen, Nat. Phys. 14, 1049 (2018).

[6] E. Abrahams and Q. Si, J. Phys.: Condens. Matter 23, 223201 (2011); J. A. N. Bruin, H. Sakai, R. S. Perry, and A. P. Mackenzie, Science 339, 804 (2013); J. Vučičević, D. Tanasković, M. J. Rozenberg, and V. Dobrosavljević, Phys. Rev. Lett. 114, 246402 (2015); J. Wang, Y.-Y. Chang, C.-Y. Mou, S. Kirchner, S. Paschen, and C.-H. Chung, arXiv:1901.10411.
[7] See T. Hu, Y. Liu, H. Xiao, G. Mu, and Y.-F. Yang, Sci. Rep. 7 , 9469 (2017), and references therein.

[8] M. Blake, R. A. Davison, and S. Sachdev, Phys. Rev. D 96, 106008 (2017).

[9] Y. Cao, D. Chowdhury, D. Rodan-Legrain, O. Rubies-Bigordà, K. Watanabe, T. Taniguchi, T. Senthil, P. Jarillo-Herrero, arXiv:1901.03710; H. Polshyn, M. Yankowitz, S. Chen, Y. Zhang, K. Watanabe, T. Taniguchi, C. R. Dean, and A. F. Young, arXiv:1902.00763.

[10] H. Okabe, N. Takeshita, K. Horigane, T. Muranaka, and J. Akimitsu, Phys. Rev. B 81, 205119 (2010).

[11] J. P. Sun, K. Matsuura, G. Z. Ye, Y. Mizukami, M. Shimozawa, K. Matsubayashi, M. Yamashita, T. Watashige, S. Kasahara, Y. Matsuda, J. Q. Yan, B. C. Sales, Y. Uwatoko, J. G. Cheng, and T. Shibauchi, Nat. Commun. 7, 12146 (2016). 
[12] J. P. Sun, G. Z. Ye, P. Shahi, J.-Q. Yan, K. Matsuura, H. Kontani, G. M. Zhang, Q. Zhou, B. C. Sales, T. Shibauchi, Y. Uwatoko, D. J. Singh, and J.-G. Cheng, Phys. Rev. Lett. 118, 147004 (2017).

[13] K. Matsuura, Y. Mizukami, Y. Arai, Y. Sugimura, N. Maejima, A. Machida, T. Watanuki, T. Fukuda, T. Yajima, Z. Hiroi, K. Y. Yip, Y. C. Chan, Q. Niu, S. Hosoi, K. Ishida, K. Mukasa, S. Kasahara, J.-G. Cheng, S. K. Goh, Y. Matsuda, Y. Uwatoko, and T. Shibauch, Nat. Commun. 8, 1143 (2017).

[14] S. Medvedev, T. M. McQueen, I. A. Troyan, T. Palasyuk, M. I. Eremets, R. J. Cava, S. Naghavi, F. Casper, V. Ksenofontov, G. Wortmann, and C. Felser, Nat. Mater. 8, 630 (2009).

[15] R. S. Kumar, Y. Zhang, S. Sinogeikin, Y. Xiao, S. Kumar, P. Chow, A. L. Cornelius, and C. Chen, J. Phys. Chem. B 114, 12597 (2010).

[16] V. Svitlyk, M. Raba, V. Dmitriev, P. Rodière, P. Toulemonde, D. Chernyshov, and M. Mezouar, Phys. Rev. B 96, 014520 (2017).

[17] C.-W. Luo, P. C. Cheng, S.-H. Wang, J.-C. Chiang, J.-Y. Lin, K.-H. Wu, J.-Y. Juang, D. A. Chareev, O. S. Volkova, and A. N. Vasiliev, Quantum Mater. 2, 32 (2017).

[18] Q. Wang, Y. Shen, B. Pan, Y. Hao, M. Ma, F. Zhou, P. Steffens, K. Schmalzl, T. R. Forrest, M. Abdel-Hafiez, X. Chen, D. A. Chareev, A. N. Vasiliev, P. Bourges, Y. Sidis, H. Cao, and J. Zhao, Nat. Mater. 15, 159 (2016).

[19] K. Kothapalli, A. E. Böhmer, W. T. Jayasekara, B. G. Ueland, P. Das, A. Sapkota, V. Taufour, Y. Xiao, E. Alp, S. L. Bud'ko, P. C. Canfield, A. Kreyssig, and A. I. Goldman, Nat. Commun. 7, 12728 (2016).

[20] B. W. Lebert, V. Balédent, P. Toulemonde, J. M. Ablett, and J.-P. Rueff, Phys. Rev. B 97, 180503(R) (2018).

[21] T. Terashima, N. Kikugawa, A. Kiswandhi, D. Graf, E.-S. Choi, J. S. Brooks, S. Kasahara, T. Watashige, Y. Matsuda, T. Shibauchi, T. Wolf, A. E. Böhmer, F. Hardy, C. Meingast, H. v. Löhneysen, and S. Uji, Phys. Rev. B 93, 094505 (2016).

[22] See also A. B. Böhmer and A. Kreisel, J. Phys.: Condens. Matter 30, 023001 (2018), and references therein.

[23] L. Craco and S. Leoni, Sci. Rep. 7, 46439 (2017).

[24] S. L. Skornyakov, V. I. Anisimov, D. Vollhardt, and I. Leonov, Phys. Rev. B 97, 115165 (2018).

[25] G. Kotliar, S. Y. Savrasov, K. Haule, V. S. Oudovenko, O. Parcollet, and C. A. Marianetti, Rev. Mod. Phys. 78, 865 (2006).

[26] L. Craco and S. Leoni, Mater. Res. Express 1, 036001 (2014).

[27] A. Yamasaki, Y. Matsui, S. Imada, K. Takase, H. Azuma, T. Muro, Y. Kato, A. Higashiya, A. Sekiyama, S. Suga, M. Yabashi, K. Tamasaku, T. Ishikawa, K. Terashima, H. Kobori, A. Sugimura, N. Umeyama, H. Sato, Y. Hara, N. Miyagawa, and S. I. Ikeda, Phys. Rev. B 82, 184511 (2010); M. D. Watson, S. Backes, A. A. Haghighirad, M. Hoesch, T. K. Kim, A. I. Coldea, and R. Valentí, ibid. 95, 081106(R) (2017).

[28] L. Craco and S. Leoni, Europhys. Lett. 92, 67003 (2010).

[29] L. Craco, M. S. Laad, and S. Leoni, J. Phys.: Conf. Ser. 487, 012017 (2014).

[30] O. K. Andersen, Phys. Rev. B 12, 3060 (1975).
[31] L. Craco, Phys. Rev. B 77, 125122 (2008).

[32] N. Dasari, W. R. Mondal, P. Zhang, J. Moreno, M. Jarrell, and N. S. Vidhyadhiraja, Eur. Phys. J. B 89, 202 (2016).

[33] M. Hirayama, T. Miyake, and M. Imada, Phys. Rev. B 87, 195144 (2013).

[34] A. van Roekeghem, L. Vaugier, H. Jiang, and S. Biermann, Phys. Rev. B 94, 125147 (2016).

[35] M. S. Laad, B. Freelon, and L. Craco, arXiv:1805.09628.

[36] K. Haule, J. H. Shim, and G. Kotliar, Phys. Rev. Lett. 100, 226402 (2008); see also L. Craco, M. S. Laad, S. Leoni, and H. Rosner, Phys. Rev. B 78, 134511 (2008).

[37] See J. Fink, J. Nayak, E. D. L. Rienks, J. Bannies, S. Wurmehl, S. Aswartham, I. Morozov, R. Kappenberger, M. A. El Ghazali, L. Craco, H. Rosner, C. Felser, and B. Büchner, Phys. Rev. B 99, 245156 (2019), and references therein.

[38] A. Georges, G. Kotliar, W. Krauth, and M. J. Rozenberg, Rev. Mod. Phys. 68, 13 (1996).

[39] A. Tamai, A. Y. Ganin, E. Rozbicki, J. Bacsa, W. Meevasana, P. D. C. King, M. Caffio, R. Schaub, S. Margadonna, K. Prassides, M. J. Rosseinsky, and F. Baumberger, Phys. Rev. Lett. 104, 097002 (2010).

[40] C. M. Varma, P. B. Littlewood, S. Schmitt-Rink, E. Abrahams, and A. E. Ruckenstein, Phys. Rev. Lett. 63, 1996 (1989).

[41] L. Craco, M. S. Laad, and S. Leoni, Phys. Rev. B 84, 224520 (2011); see also B. Freelon, Y. H. Liu, J.-L. Chen, L. Craco, M. S. Laad, S. Leoni, J. Chen, L. Tao, H. Wang, R. Flauca, Z. Yamani, M. Fang, C. Chang, J.-H. Guo, and Z. Hussain, ibid. 92, 155139 (2015).

[42] S. Biermann, L. de' Medici, and A. Georges, Phys. Rev. Lett. 95, 206401 (2005); see also L. Craco, Phys. Rev. B 59, 14837 (1999).

[43] P. W. Anderson and H. Hasegawa, Phys. Rev. 100, 675 (1955).

[44] R. Yu and Q. Si, Phys. Rev. B 96, 125110 (2017); L. Craco, M. S. Laad, and S. Leoni, J. Phys.: Condens. Matter. 26, 145602 (2014); see also L. Craco and S. Leoni, Phys. Rev. B 98, 195107 (2018), and references therein.

[45] A. Hackl and M. Vojta, New J. Phys. 11, 055064 (2009).

[46] K. Haule and G. Kotliar, New J. Phys. 11, 025021 (2009).

[47] S. Cai, J. Guo, V. A. Sidorov, Y. Zhou, H. Wang, G. Lin, X. Li, Y. Li, K. Yang, A. Li, Q. Wu, J. Hu, S. K. Kushwaha, R. J. Cava, and L. Sun, Quantum Mater. 3, 62 (2018).

[48] Y. M. Dai, H. Miao, L. Y. Xing, X. C. Wang, C. Q. Jin, H. Ding, and C. C. Homes, Phys. Rev. B 93, 054508 (2016); see also W. Li, H. Ding, P. Deng, K. Chang, C. Song, K. He, L. Wang, X. Ma, J.-P. Hu, X. Chen, and Q.-K. Xue, Nat. Phys. 8, 126 (2012); S. He, J. He, W. Zhang, L. Zhao, D. Liu, X. Liu, D. Mou, Y.-B. Ou, Q.-Y. Wang, Z. Li, L. Wang, Y. Peng, Y. Liu, C. Chen, L. Yu, G. Liu, X. Dong, J. Zhang, C. Chen, Z. Xu et al., Nat. Mater. 12, 605 (2013); X. Chen, P. Lu, X. Wang, Y. Zhou, C. An, Y. Zhou, C. Xian, H. Gao, Z. Guo, C. Park, B. Hou, K. Peng, X. Zhou, J. Sun, Y. Xiong, Z. Yang, D. Xing, and Y. Zhang, Phys. Rev. B 96, 165123 (2017).

[49] J. Bardeen, Phys. Rev. Lett. 1, 399 (1958). 